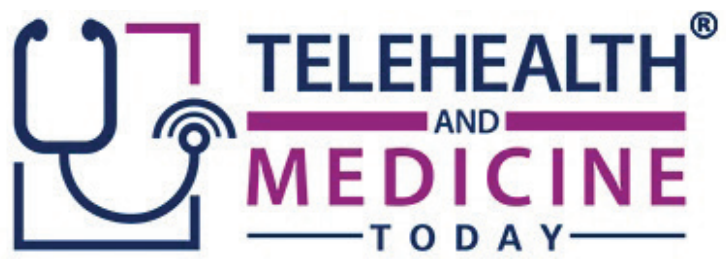

\title{
Feasibility and Effectiveness of Mobile App for Active Case Finding for Tuberculosis in India
}

Weijia Zhang ${ }^{1}$, Mariam E Dogar ${ }^{2}$, Monika Jain ${ }^{3,4}$, Edwin Rodriges ${ }^{5}$, Sangeeta Pathak $^{4}$, Salil Bhargava ${ }^{4,6}$, Amar Gupta ${ }^{7,8}$, Manoj Jain ${ }^{4,9}$

Affiliations: ${ }^{1}$ Wellesley College, Wellesley, MA, USA; ${ }^{2}$ Massachusetts Institute of Technology (MIT), Cambridge, MA, USA; ${ }^{3} \mathrm{TSU}$ Quillen College of Medicine, Mountain Home, TN, USA; ${ }^{4}$ Collaborative to Eliminate Tuberculosis in India (CETI), Indore, India; ${ }^{5} \mathrm{EC}$ Digital Services, India; ${ }^{\mathrm{MGM}}$ Medical College, Indore, India; ${ }^{7} \mathrm{Computer}$ Science and Artificial Intelligence Labs (CSAIL), Institute of Medical Engineering and Science (IMES), ${ }^{8}$ Department of Electrical Engineering and Computer Science, MIT, Cambridge, MA, USA; ${ }^{9}$ Rollins School of Public Health, Emory University, Atlanta, GA, USA

Corresponding Author: Weijia Zhang, Email: wzhang0333@gmail.com

Keywords: Active Case Finding, Telemedicine, Tuberculosis.

Section: Original Research

Background: Tuberculosis (TB) is an infectious disease with 2.8 million cases and 480,000 deaths each year in India. The city of Indore alone with a population of 3.5 million had 7,839 identified TB cases in 2017. However, about two to three thousand additional cases remain unidentified per district officials. The unidentified cases lead to an endemic TB and hamper the efforts of organizations such as The Collaborative to Eliminate TB from India (CETI) to reduce the incidence of TB with the method of Active Case Finding (ACF). ${ }^{1}$ Previously, 1,332 mobile apps attempted to use technology to overcome the challenge of unreported TB patients in Indian slum areas due to the inaccurate, lost, or unhelpful data collected in $A C F$; yet the existing apps for TB prevention and treatment possessed minimal functionality. Over a period of 3 months, the CETI developed a mobile data collection app to generate a TB diagnostic survey and to collect data from patient registration form.

Methods: To study the feasibility and effectiveness of the app, a pilot survey was conducted of 163,496 homes covering a population of 828,020 in the slum areas of Indore and Bhopal.

Findings: Between the years of 2018 and 2019, 14,349 pulmonary suspected cases and 4,357 extra pulmonary suspected cases of TB were identified. Among the total of 18,706 cases identified, 7,756 patients (48.1\%) had low-grade 
fever for over 2 weeks, 6,331 patients (39.2\%) had persistent cough for more than 2 weeks, 7,693 patients (47.7\%) had weight loss, and 251 patients (1.6\%) had cough with blood.

Interpretation: This pilot experience shows that an app is a useful tool for TB case recording and follow-up in the field. Further training of the health workers, and more widespread availability and ease of use of mobile phones will be necessary.

\section{Funding: unidentified.}

\section{BACKGROUND}

Tuberculosis (TB) is an infectious disease caused by Mycobacterium tuberculosis and spreads through the air when a person with active TB speaks, coughs, and sneezes. TB leads to 2.8 million cases and 480,000 deaths each year in India as reported by the Government of India annual report in 2018. ${ }^{2}$ Indore, a city in central India, has about 3.5 million people and had 7,839 notified cases of TB in 2017, as announced by the report. ${ }^{2}$ As per the district officials, an estimated two to three thousand additional cases were neither identified nor notified (in a personal communication with District TB Officer, Indore). Unidentified cases lead to an endemic TB and hamper the efforts to reduce the incidence of TB. Even when patients with symptoms of low-grade fever and persistent cough are identified in the field, less than $50 \%$ of them follow up with further testing. ${ }^{3}$

The Collaborative to Eliminate TB from India (CETI), an NGO based in Indore, employs community health workers for the purpose of Active Case Finding (ACF) to identify, diagnose, and follow up on suspected cases of TB. ${ }^{4}$ Since the challenge of unreported and untreated TB patients in Indian slum areas is exacerbated by inaccurate, lost, or unhelpful data collected during ACF, CETI applies technology to enhance the collection and processing of data in the field. Since a 2016 survey of 1,332 potential "apps identified for TB prevention and treatment had minimal functionality," CETI decided to use the Dimagi CommCare platform to develop an app for ACF for TB. ${ }^{5}$ The pilot implementation experience is described in this article.

\section{METHODS}

In the app, the Diagnostic TB Survey module collects all the information needed to identify a suspected TB patient and is equipped with an algorithm to alert the surveyor of a potential TB suspect case. Patients suspected to have TB are then registered by the patient registration form, which records all the information that a CETI worker needs to follow up with the patients regarding their clinical results, further pathology tests, the status of the medical treatments, and awareness education and TB counseling. If on further testing, persons are identified as having TB, they are referred to Revised National TB Control Program (RNTCP) for treatment. Hence, the CETI assists RNTCP in identifying TB cases and the TB patients in going through proper treatments. Moreover, the app aids CETI workers on tasks related to their daily ACF work.

To study the feasibility and effectiveness of the app, a survey was conducted of 163,496 homes covering a population of 828,020 in the slum areas of Indore and Bhopal. The patients participating in the survey were selected based on the contact made by the ACF teams in the regions of Indore, Madhya Pradesh and its outskirts, who talked to citizens about themselves and those living in their home. Participants were first asked if 
they or their family members were experiencing any symptoms of $\mathrm{TB}$, and then they were provided a follow-up information based on their responses. If patients were concluded to be a suspected TB case, they were given a sputum cup to collect sputum for further testing and were recommended to come to a local hospital for a chest X-ray for further evaluation.

\section{FINDING}

Between the years of 2018 and 2019, $1,370,623$ persons $(N)$ were reviewed during house visits, including the cities of Indore (867,156 persons) and Bhopal $(191,896$ persons). A total of 18,706 cases $(n)(1.36 \%)$ were reported with presumptive TB, among which 14,349 (76.7\%) pulmonary suspected cases and 4,357 (23.3\%) extra pulmonary suspected cases of TB were identified. Among these suspected cases, 8,473 (45.3\%) patients were female, and 10,176 (54.4\%) patients were male.

Out of the 16,136 cases that were obtained with data of present symptoms of TB, 7,756 patients (39.2\%) had low-grade fever for over 2 weeks, 6,331 patients $(72.5 \%)$ had persistent cough for more than 2 weeks, 7,693 patients (47.7\%) had weight loss, and 251 patients $(1.6 \%)$ had cough with blood. The recorded data were used to follow up patients by in person and by phone. In addition, supervisors not in the field were actively able to monitor the case load and follow-up rates.

\section{DISCUSSION}

The mobile app was developed on CommCare mobile data collection platform by the CETI over a period of 3 months. ${ }^{5}$ In the first phase, survey forms and patient forms were generated and used in the field as pilot. In the second phase, forms were modified as per the mobile worker requirement, such as listing only the nearest DMCs (rather than the full list) and patient's geolocation mapping to enable the field sputum collector to easily reach the location of a patient who suspected of TB. The CETI is currently working on the third phase, wherein the followups can be done using the app.

The app improves the quality of diagnostic decision, collects useful information, and keeps a secure record of all the data collected. The app has phased out the paper-based ACF, which took days to process and was prone to being lost, to a one-time process completed within minutes using a mobile phone. Further, the app incorporates an expanded list of survey questions to ensure more accurate diagnosis, along with the technique of educational and motivational interviewingwhere the interviewer informs the interviewee

Table 1. Population characteristics

\begin{tabular}{|l|c|}
\hline Population, $N$ & $1,370,623$ \\
\hline Total presumptive TB patients (both pulmonary and extra pulmonary), $n$ & 18,706 \\
\hline Male & 10,176 \\
\hline Female & 8,473 \\
\hline Sputum collected & 1,457 \\
\hline X-rays done & 2,242 \\
\hline Phone numbers obtained & $99.89 \%$ \\
\hline
\end{tabular}




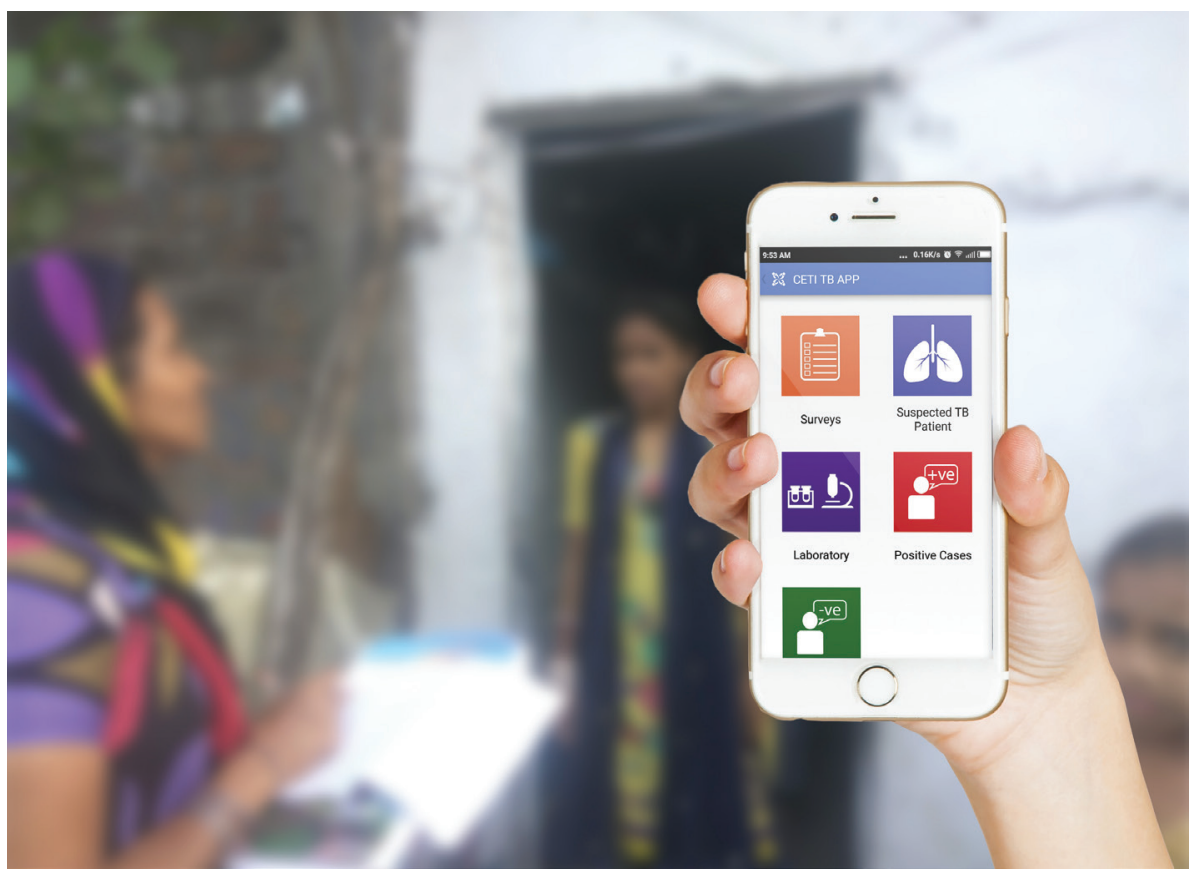

Figure 1-CETI TB APP homepage.

about the basic symptoms of TB and to seek care if symptoms appear. In areas of poor internet connectivity, the app saves data on the mobile phone for subsequent upload to the server. The geographic information of the registered patients alerts the CETI about locations with higher incidence of TB.

One key observation for conducting the surveys is that there is a general lack of understanding regarding what $\mathrm{TB}$ is and how it is spread in the general population. Informational pamphlets were distributed by ACF volunteers, and CETI had organized smaller educational events such as 1-day camps, talks at local schools, and the Lambhi Khasi Dheeme Bhukar (Long Cough, Low Fever) initiative. However, the general feel from the community is that they are not quite ready to make the changes needed to decrease TB cases. There are problems in the infrastructure of the healthcare system, which dampens the response and acceptance by patients. The CETI is currently working to institute a change in Indore similar to the Swacch Bharat (Clean India) campaign across all over India, so that TB can be treated with the same level of seriousness and dedication by both the citizens and the Indian government.

\section{CONCLUSIONS}

This pilot experience shows that a mobile app can be a useful tool for TB case recording and follow-up in the field. For case identification, the app may be useful but further training of health workers and more widespread availability and ease of use of mobile phones will be necessary.

Overall the app provides a new approach to $\mathrm{ACF}$, leveraging the advantages of technology to enable health workers to interact more effectively with potential TB patients, and provide efficiency in $\mathrm{ACF}$ and follow-up treatments. 


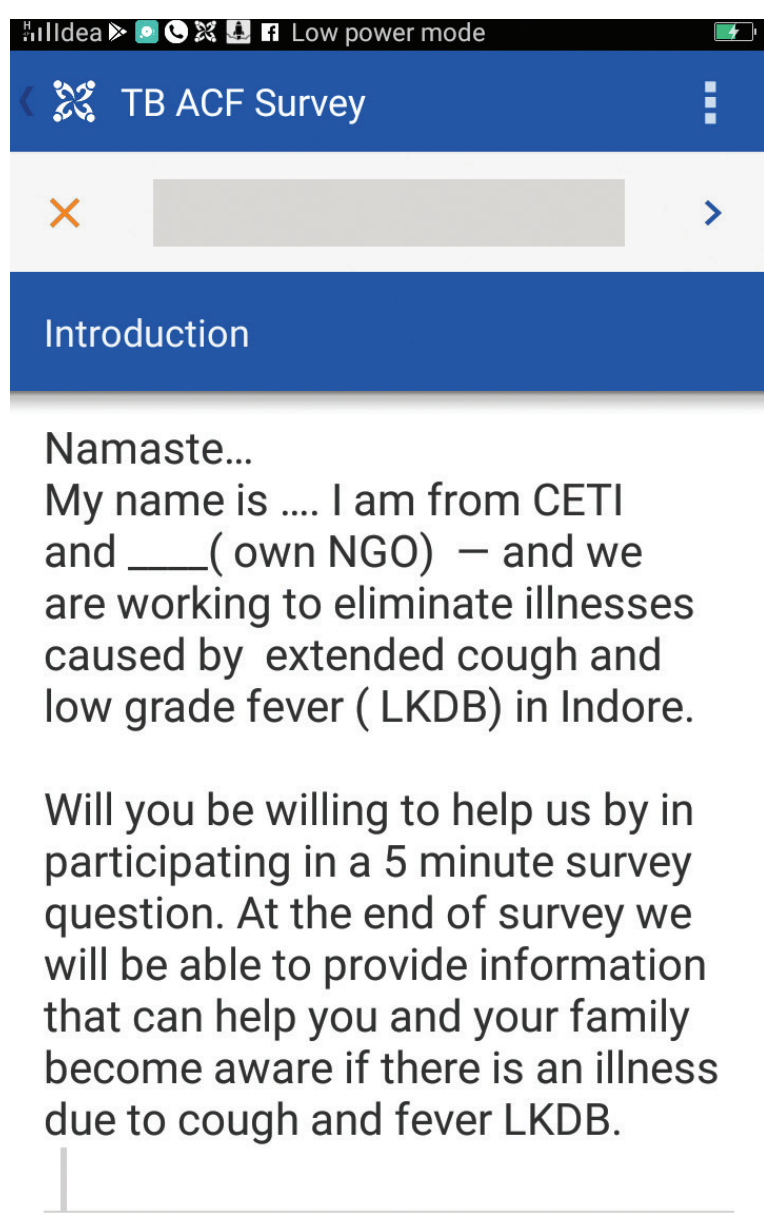

Figure 2-CETI TB APP introduction page.

Conflict of interests: Apart from the disclosure in the funding statement below, the authors confirm that there are no known conflicts of interest associated with this publication and that there has been no financial support for this work that could have influenced its outcome. Dr Amar Gupta has served as an advisor to MITRE for technical exchange meeting involving US Federal Government, and paid as independent consultant, a Paid Keynote Address for Artificial Intelligence in Healthcare: The Role of Medical Boards; Dr Gupta was paid for by McDermott Will \& Emery LLP, consultant to Walmart Health for Telemedicine for Business
Strategy, paid for by Walmart; Dr Gupta is a lead researcher on telemedicine interactions and face-to-face interactions in critical care medicine and other clinical specialties: Enhancing Effectiveness of Virtual Teams thru Systems Engineering" project sponsored by Philips at MIT and receiving funding as salary via MIT; Dr Gupta serves as a consultant to the United Technology Corporation on issues related to AI, not healthcare, and is part of proceed received for MIT Hacking Machine and King Abdulaziz City for Science and Technology used to support Dr Gupta's research and team at MIT.

Contributors: W.Z., M.E.D., M.J., E.R., S.P., M.J., and A.G. made substantial contributions to the conception or design of the work; S.P.E.R. and M.J. contributed to the acquisition; E.R. and M.J. contributed to the interpretation of the data; W.Z., M.E.D., M.J., and A.G. contributed to drafting the work or revising it; W.Z., M.E.D., M.J., E.R., S.P., M.J., and A.G. contributed to the final approval of the version to be published.

Funding Statement: Dr Amar Gupta is an employee at MIT and was partly funded from a research contract signed by Philips.

\section{REFERENCES}

1. Collaboration for Elimination of TB (CETI). Collaboration to eliminate tuberculosis in India: TB free India [homepage on the Internet]. CETI; 2020 [cited 2020 Jun 26]. Available from: https:// www.tbfree.org/

2. Ministry of Health \&amp; Family Welfare - Government of India. TB India report 2018 [Internet]. [cited 2020 Jun 26]. 
Available from: https://tbcindia.gov.in/ showfile.php?lid=3314

3. CETI experience.

4. Active case finding: Systematic screening for active tuberculosis [Internet]. World Health Organization; 2015 [cited 2020 Jun 26]. Available from: https://www.who.int/ tb/areas-of-work/laboratory/active-casefinding/en/

5. Iribarren SJ, Schnall R, Stone PW, Carballo-Diéguez A. Smartphone applications to support tuberculosis prevention and treatment: Review and evaluation [Internet]. JMIR mHealth and uHealth. JMIR Publications;
2016 [cited 2020 Jun 26]. Available from: https://www.ncbi.nlm.nih.gov/ pubmed/27177591

Copyright Ownership: This is an open access article distributed in accordance with the Creative Commons Attribution Non Commercial (CC BY-NC 4.0) license, which permits others to distribute, adapt, enhance this work noncommercially, and license their derivative works on different terms, provided the original work is properly cited and the use is noncommercial. See: http:// creativecommons.org/licenses/by-nc/4.0. 


\section{Appendix 1a: TB ACF Survey Questions (English)}

1. Do you or anybody in your family have any of the following symptoms:

Cough for more than 2 weeks? Cough accompanied by bleeding for more than 2 weeks? Fever for more than 2 weeks? Less fever and sweating during the night; Loss of weight?

2. Do you or anybody else in your family smoke?

3. Do you or anybody else in your family have HIV?

4. Does anybody in your family have a history of tuberculosis (TB) and been treated for it?

5. Was the treatment for anybody in your family who had TB been unsuccessful?

6. Symptoms of weight loss and fever?

7. Apart from light fever in the evening, loss of weight, and loss of appetite, do you have the feeling of water filling up or pain in any part of the body?

8. Are you or any member of your family characterized by any of the following symptoms: Stiffness in any part of the body; Loss of weight, fever, or pain in the body; Light fever in the body; Loss of weight?

9. Does anybody in your family consume alcohol?

10. Do you or anybody in your family consume opium?

11. Enter patient's mobile number, is a sputum cup given?, comments

\section{Appendix 1b: Patient Registration Form Questions (English)}

1. Name of Patient

2. Occupation

3. Mobile Number

4. Age

5. Gender

6. Case Type (Pulmonary, Extra Pulmonary, History of TB, Other)

7. Action taken? 